\title{
Le spectre de la crise financière française de 1983
}

\author{
Influences et solidarités européennes
}

\author{
Laurent Warlouzet
}

Version auteur de: Laurent Warlouzet, " Le spectre de la crise financière française de 1983. Influences et solidarités européennes", in Vingtième Siècle. Revue d'histoire, 2018/2, n० 138, pp. 93-107.

DOI : 10.3917/ving.138.0093

Cet article procède à la relecture des mécanismes qui ont conduit au tournant de la rigueur de mars 1983, dont il propose de déconstruire l'interprétation historiographique qui a jusque-là prévalu et qui insiste sur la rupture nette opérée à cette occasion. Pour Laurent Warlouzet, les décisions prises par le gouvernement français relèvent moins de l'influence néolibérale britannique que d'une convergence franco-allemande et de contraintes liées au contexte difficile dans lequel se trouve alors l'économie française.

Le «tournant de la rigueur » constitue l'expression consacrée pour désigner les décisions prises par le gouvernement français en mars 1983 : dévaluer tout en restant dans le Système monétaire européen (SME) grâce à un plan d'économies. La voie européenne promue par Jacques Delors, ministre de l'Économie et des Finances, l'emportait alors sur les tentations protectionnistes, incarnées en particulier par le ministre de l'Industrie Jean-Pierre Chevènement. L'événement a longtemps été interprété comme un tournant radical, et parfois lu comme l'adoption du néolibéralisme par le gouvernement français. On doit cette interprétation, influente, à Bruno Théret et Bruno Jobert ${ }^{1}$.

Pourtant plusieurs historiens, notamment Michel Margairaz, ont remis en cause cette notion de rupture brutale, ce dernier lui préférant l'idée de « virage », soit une inflexion dans un processus complexe, au sein duquel coexistaient plusieurs approches économiques prises

\footnotetext{
${ }^{1}$ Bruno Jobert et Bruno Théret, « France : la consécration républicaine du néolibéralisme », in Bruno Jobert (dir.), Le Tournant néolibéral en Europe, Paris, L’Harmattan, 1994, p. 21-86. Pour une analyse historique complète des différentes interprétations françaises des événements de 1983 depuis l'origine, voir Matthieu Fulla, «État de la question, historiographie », communication lors du colloque organisé par Olivier Feiertag et Michel Margairaz et intitulé « Une histoire du tournant de la rigueur », les 25 et 26 mars 2013 à Paris.
} 
en compte par le gouvernement depuis $1981^{2}$. De même, dans une brillante synthèse consacrée à la France des années 1980, Ludivine Bantigny relativise la rupture de 1983, en soulignant que la ligne de l'austérité budgétaire s'était exprimée dès l'automne $1981^{3}$. Dans sa notice pour l'Histoire mondiale de la France, François Denord reprend à son compte ces nuances en soulignant que « parler de "tournant" se révèle, à bien des égards, exagéré ${ }^{4}$ », car le débat entre les deux gauches a été constant au sein du Parti socialiste depuis les années 1970. Enfin, dans sa thèse de doctorat consacrée à la politique du travail de 1981 à 1984, Matthieu Tracol adopte la même perspective à partir d'une analyse fouillée utilisant des sources nouvelles, notamment le fonds Mauroy ${ }^{5}$.

Le poids symbolique de l'alternative de 1981 dans l'histoire de France justifie cette multiplication de travaux aux thèses convaincantes, adoptant du reste une perspective majoritairement française. Cette contribution se propose de les compléter par une perspective européenne afin d'évaluer, d'une part, l'originalité de la trajectoire française en Europe, et, d'autre part, le rôle de l'Europe dans le processus de décision, qu'il s'agisse de la Communauté économique européenne (CEE) d'alors ou des gouvernements nationaux pris individuellement ${ }^{6}$. La «contrainte extérieure », dont le poids a été souligné par l'analyse de Jean-Charles Asselain, sera ainsi incarnée par des acteurs concrets ${ }^{7}$.

L'article vise ainsi à montrer qu'un tournant véritable eu lieu en mars 1983 en termes de convergence avec les politiques économiques majoritaires au sein de la CEE, et ce du fait de la relation franco-allemande, plus que d'une pression de la Commission européenne ou de la Grande-Bretagne thatchérienne. L’engagement européen du président Mitterrand était

${ }^{2}$ Michel Margairaz, «L'impératif économique : l'ajustement périlleux entre relance, réforme et rigueur », in Serge Berstein, Pierre Milza et Jean-Louis Bianco (dir.), François Mitterrand : les années du changement, 1981 1984, Paris, Perrin, 2001, p. 333-343; Michel Margairaz, «Les décideurs de la politique économique et financière des années 1970-1980 », communication lors du colloque organisé par Olivier Feiertag et Michel Margairaz et intitulé « Une histoire du tournant de la rigueur », les 25 et 26 mars 2013 à Paris.

${ }^{3}$ Ludivine Bantigny, La France à l'heure du monde : de 1981 à nos jours, Paris, Éd. du Seuil, 2013, p. 32.

${ }^{4}$ François Denord, « 1983 : la rigueur des temps », in Patrick Boucheron (dir.), Nicolas Delalande, Florian Mazel, Yann Potin et Pierre Singaravélou (coord.), Histoire mondiale de la France, Paris, Éd. du Seuil, 2017, p. 723 .

5 Matthieu Tracol, «La Rigueur et les réformes: histoire des politiques du travail et de l'emploi du gouvernement Mauroy (1981-1984) », thèse pour le doctorat en histoire, Université Paris-I, 2015, p. 14.

${ }^{6}$ Cet article s'inscrit dans un travail plus large sur la mutation des politiques économiques ouest-européennes face à la mondialisation de 1973 à 1986 : Laurent Warlouzet, Governing Europe in a Globalizing World : Neoliberalism and its Alternatives following the 1973 Oil Crisis, Londres, Routledge, 2018.

${ }^{7}$ Voir l'article de Jean-Charles Asselain dans ce numéro. 
antérieur à $1983^{8}$, mais il n'était pas visible du fait de l'originalité de la trajectoire économique française en Europe. Ce hiatus disparaît en mars 1983, ouvrant la voie à la renaissance d'un couple franco-allemand efficace. Par ailleurs, cette contribution s'attache à inscrire le «tournant de la rigueur » dans la transition des politiques économiques et sociales occidentales, d'une dominante keynésienne à une optique plus libérale. Si la politique néolibérale se définit par la volonté de s'attaquer à l'État-providence, à l'image de la politique thatchérienne d'alors, alors la crise de 1983 ne donne pas lieu à un tournant néolibéral, mais plutôt à une convergence vers des politiques de libre-marché adaptées au défi de la mondialisation, sans être incompatibles avec des politiques sociales ${ }^{9}$.

À l'appui de cette thèse ont été mobilisées des archives permettant d'étudier les quatre acteurs principaux, les gouvernements français, britannique et allemand (y compris les archives de la Bundesbank), ainsi que les archives de la Confédération européenne des syndicats (CES), afin d'apporter un éclairage sur le débat transnational au sein de la gauche européenne.

Tout d'abord, l'article insiste sur l'originalité de la relance conduite par le président Mitterrand dans l'Europe de la période 1981-1983. Ensuite, il souligne l'ampleur de la crise financière que connaît le gouvernement français en 1983. Enfin, il affirme le caractère déterminant de la relation franco-allemande dans la résolution de la crise.

\section{L'originalité de la relance Mitterrand en Europe}

En 1981, l'arrivée au pouvoir de François Mitterrand produit une rupture majeure sur le plan politique, avec le retour de la gauche au pouvoir après vingt-trois années d'absence. Les réformes sociales sont très nombreuses, avec une augmentation des salaires et des minima sociaux, une réduction du temps de travail (cinquième semaine de congés payés annuels et passage du temps de travail hebdomadaire de quarante à trente-neuf heures) et une relance des

\footnotetext{
${ }^{8}$ Georges Saunier, « Le gouvernement français et les enjeux économiques européens à l'heure de la rigueur, 1981-1984 », in Éric Bussière, Michel Dumoulin et Sylvain Schirmann (dir.), Milieux économiques et intégration européenne au XXe siècle : la relance des années quatre-vingts (1979-1992), Paris, CHEFF, 2007, p. 109-146.

${ }^{9}$ Sur cette distinction entre politique de libre-marché et politique néolibérale, la seconde étant une variante radicalisée de la première caractérisée par la volonté de réduire l'État-providence, voir L. Warlouzet, Governing Europe in a Globalizing World..., Londres, Rouledge, 2017, p. 7-8 et 32-35. Cette définition est sensiblement différente de celle adoptée dans une approche plus proche de l'histoire des idées : François Denord, « Néo-
} 
embauches dans la fonction publique. La logique est sociale mais aussi économique car ces réformes doivent soutenir une politique de relance qui vise à résorber le chômage, en constante progression depuis le premier choc pétrolier de 1973. Avant cette date, le taux de chômage était inférieur à $3 \%$ de la population active alors qu'il dépasse $7 \%$ en 1981 et $8 \%$ en 1982.

En complément, les nationalisations doivent servir à réorganiser le tissu industriel français autour de champions nationaux spécialisés, alors que les groupes privés s'épuisent en concurrences intestines ruineuses. Pour les nouveaux responsables socialistes, le contreexemple est donné par l'approche de l'équipe précédente, celle du président Valéry Giscard d'Estaing et du Premier ministre Raymond Barre, qui ont prétendu mener une politique libérale tout en aidant massivement des entreprises privées finalement perçues comme inefficaces, en particulier dans la sidérurgie ${ }^{10}$. Le gouvernement britannique travailliste n'a-til pas nationalisé en 1977 deux secteurs en déshérence, la construction navale (British Shipbuilding) et l'industrie automobile (British Leylands) ? L'intervention publique paraît ainsi constituer, pour les responsables socialistes, non seulement un choix idéologique, mais aussi une solution efficace face à un patronat incompétent.

Par ailleurs, il faut noter qu'à l'échelle européenne, les responsables socialistes disposaient d'une certaine marge de manœuvre. Ainsi, une enquête française conduite en 1979, dans le cadre du débat européen sur l'aménagement du temps de travail, révèle que la durée annuelle du travail en France était, en 1977, de 1900 heures contre 1820 heures au Royaume-Uni, 1750 h en République fédérale d'Allemagne (RFA) et en Belgique, 1540 heures en Italie et, à titre de comparaison, 1700 heures aux États-Unis et 2100 heures au Japon ${ }^{11}$. Parmi les pays voisins, seuls les Pays-Bas connaissent une durée légèrement supérieure, avec 1940 heures De même, en termes de déficits, la gestion prudente de l'austère Raymond Barre a permis de préserver des marges de manœuvre, et ce malgré une mini-relance à l'extrême fin de son mandat. En 1979 et en 1980, le déficit de l'administration centrale est légèrement inférieur en France par rapport à l'Allemagne de l'Ouest (voir le document 1). Ainsi, au

libéralisme et "économie sociale de marché": les origines intellectuelles de la politique européenne de la concurrence (1930-1950)», Histoire, économie et société, 27 (1), 2008, p. 23-34.

${ }^{10}$ Voir le constat globalement critique mais nuancé de la Commission du bilan présidée par François BlochLainé : Commission du bilan, La France en mai 1981 : forces et faiblesses, Paris, La Documentation française, 1981, p. 128-134, notamment p. 131 pour la sidérurgie.

11 Archives nationales (AN) françaises, 5AG3/915, note du conseiller technique Olivier Fouquet à la présidence de la République, 9 mars 1979 ; note du ministère du Travail, 6 mars 1979. 
tournant de 1980, le gouvernement français paraît plus vertueux que son homologue allemand, une situation rare dans l'après-guerre ${ }^{12}$. La Bundesbank se plaint encore des déficits allemands au début de l'année $1981^{13}$. Au-delà de ces deux pays, les déficits sont plus importants encore en Grande-Bretagne, aux États-Unis et surtout en Italie.

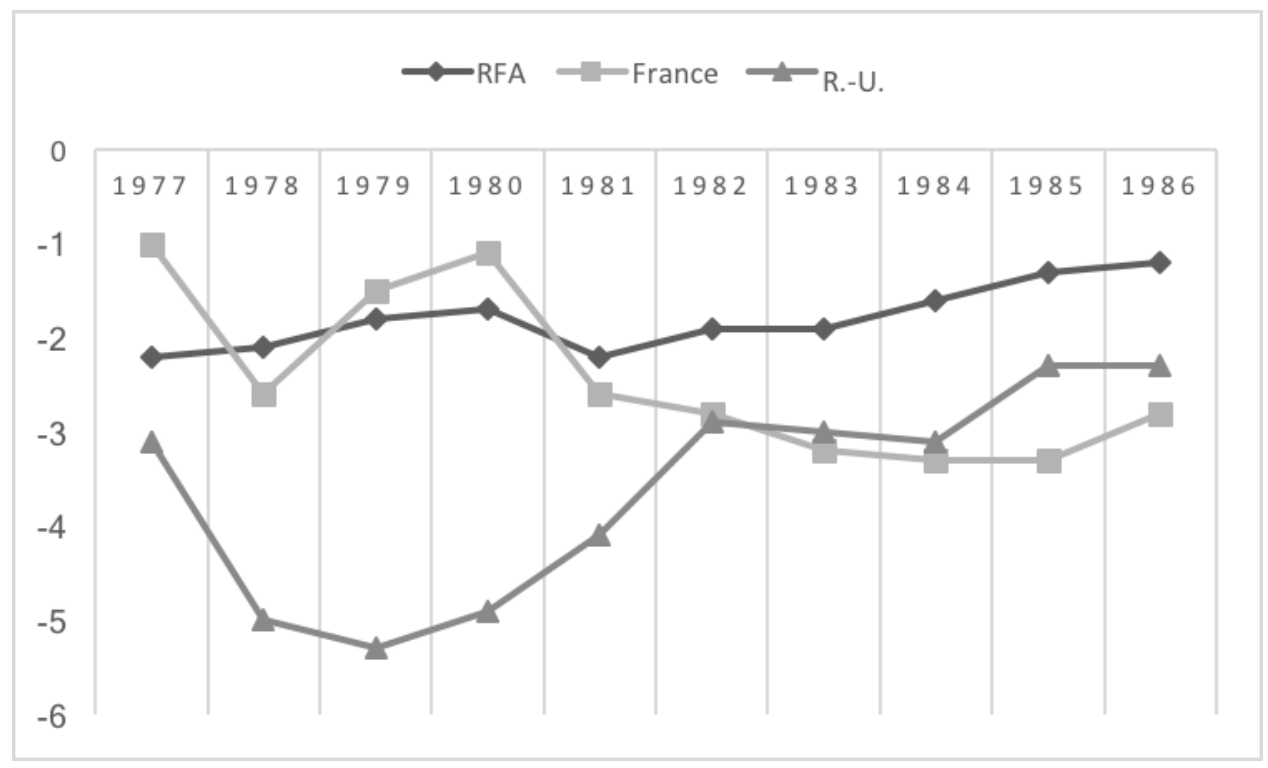

1. Solde des administrations publiques en pourcentage du PNB ${ }^{14}$

L'intensité de la relance Mitterrand fait débat. Elle a certes été significative, mais moins importante financièrement que la relance Chirac de 1975. Cependant, à la différence de cette dernière, elle crée des charges permanentes par le biais des réformes sociales, des nationalisations et de la création d'emplois publics ${ }^{15}$. Le fait que la relance ait été consacrée aux trois quarts à la consommation, et seulement pour un quart à l'investissement ${ }^{16}$, est aussi l'objet de débat. Les responsables socialistes comptaient sur une reprise économique rapide mais, dès juillet 1981, l'OCDE revoit à la baisse ses prévisions ${ }^{17}$. Surtout, la relance française intervient après huit années de crise économique qui ont démontré le caractère structurel de la

${ }^{12}$ Sur les difficultés financières allemandes, voir L. Warlouzet, Governing Europe in a Globalizing World..., op. cit., p. 20 et 145 .

13 Archives de la Bundesbank, B 330/11165, réunion du Zentralbankrats du 19 février 1981.

${ }^{14}$ Source pour la période 1977-1982, FMI, Rapport annuel 1984, Washington, 1985, p. 5 ; pour la période 1983-1986, FMI, Rapport annuel 1987, Washington, 1988, tableau 1.

15 Jean-Charles Asselain, «L'expérience socialiste face à la contrainte extérieure », in Serge Berstein et al. (dir.), François Mitterrand..., op. cit., p. 400 et 429 ; voir aussi l'article publié dans ce numéro.

${ }^{16}$ Robert Salais, « De la relance à la rigueur », in Serge Berstein et al. (dir.), François Mitterrand..., op. cit., p. $476-505$.

${ }^{17}$ Claude Beaud, Chocs et entrechocs de l'économie mondiale, 1973-1987, Paris, Sedes, 1988, p. 144. 
crise. La dépression est encore amplifiée par le deuxième choc pétrolier de la fin de l'année 1979 qui relance l'inflation et l'augmentation du chômage, tout en diminuant la croissance. Son impact est magnifié par la décision du nouveau président de la Réserve fédérale étatsunienne, Paul Volcker, prise en 1979, d'augmenter massivement les taux d'intérêt afin de briser l'inflation. Tous les autres pays sont obligés de suivre, ce qui contribue à dégrader la conjoncture. Alors que le recours à la relance keynésienne semblait un réflexe naturel dans l'Europe de 1975, il ne l'est plus en 1981, comme le montrent les exemples des politiques conduites par les trois pays grands voisins, Grande-Bretagne, Allemagne et Italie, et comme le suggèrent les réflexions de la Confédération européenne des syndicats.

Le Royaume-Uni offre le contraste le plus saisissant avec la France. Margaret Thatcher, devenue Premier ministre en 1979, impose une politique d'austérité budgétaire radicale, et de taux d'intérêt élevés afin de lutter contre l'inflation. Cette politique entraîne des protestations massives en 1980-1981, y compris de la part du patronat qui se plaint de taux d'intérêt trop élevés, et suscite de sérieux doutes au sein même du parti conservateur ${ }^{18}$. En effet, les résultats ne sont pas au rendez-vous à court terme, en termes de croissance, de chômage, ni d'inflation, avec un pic à 21,9\% en mai 1980 (en rythme annuel). Dans le domaine de la politique industrielle, la mutation est plus progressive car les privatisations massives n'interviennent qu'après sa réélection de 1983.

En Allemagne, le chancelier social-démocrate allemand Helmut Schmidt, issu de l'aile modérée du SPD, associe l'impératif de la préservation de la remarquable compétitivité des entreprises allemandes, par un contrôle de l'inflation et des équilibres budgétaires, à des relances keynésiennes ciblées depuis 1975 qui portent en général plus sur l'investissement que sur la consommation ${ }^{19}$. Cette politique s'interrompt aux lendemains du second choc pétrolier, qui provoque une récession très aiguë et un déficit de la balance des paiements. La dégradation de la conjoncture provoque la publication d'un rapport alarmiste sur le déficit budgétaire, qui pousse Schmidt à lancer en décembre 1981 un vaste programme

${ }^{18}$ Keith Middlemas, Power, Competition and the State, vol. 3 : The End of the Postwar Era : Britain since 1974, Basingstoke, Macmillan, 1991, p. 350 ; David Parker, The Official History of Privatisation, vol. 1 : 19791987, Londres, Routledge, 2009, p. 70.

19 Tim Schanetzky, Wirtschaftspolitik, Expertise und Gesellschaft in der Bundesrepublik 1966 bis 1982 , Berlin, Akademie Verlag, 2007, p. 273; Wolfgang Neumann et Henrik Uterwedde, Industriepolitik: ein deutsch-französischer Vergleich, Leverkusen, Leske, 1986, p. 66. 
d'assainissement des finances publiques ${ }^{20}$. La crise économique et les divisions entre le SPD et le FDP provoquent finalement la chute du gouvernement et l'arrivée au pouvoir du gouvernement Kohl, à la tête d'une nouvelle coalition CDU - CSU-FPD. Face à l'ampleur du déficit budgétaire, le gouvernement prend immédiatement des mesures d' « urgence » fondées sur des économies budgétaires ciblées. Toutefois, la rupture est plus forte dans le discours que dans les faits. Le gouvernement prévoit toujours quelques mesures de soutien aux investissements et à la construction immobilière à la fin de l'année $1982^{21}$. Si la transition est progressive, Kohl poursuit une politique de réduction du déficit public, qui passe de 3,7\% PIB en 1981 à un peu plus de $1 \%$ en $1985^{22}$.

En dehors des gouvernements, les réflexions au sein de la CES convergent également vers l'idée que toute tentative de relance keynésienne de manière unilatérale et centrée sur la consommation dans un monde ouvert serait illusoire. En 1977, la CES s'exprime ainsi : « La CES reconnaît que l'interdépendance des économies de la Communautés est maintenant telle que la plupart des pays ont moins de possibilité de décider seuls des politiques économiques de management de la demande qu'ils entendent poursuivre, et que de toute façon ces politiques de management de la demande ne parviendront pas à réstaurer le plein emploi dans un délai raisonnable sans effets inacceptables sur l'inflation ou sur la balance des paiements ${ }^{23}$. » La CES réaffirme cette position en 1980, tout en encourageant des mesures ciblées, notamment à destination des plus bas salaires ${ }^{24}$.

Pour autant, il ne faut pas considérer les années 1981-1983 comme dominée par le paradigme néolibéral. Les résultats macroéconomiques britanniques restent extrêmement

${ }^{20}$ Hélène Miard-Delacroix, Le Défi européen de 1963 à nos jours, Villeneuve-d'Ascq, Septentrion, 2011, p. 26 et 65 .

${ }^{21}$ Reimut Zohlnhöfer, Die Wirstchaftspolitik der Ära Kohl : eine Analyse der Sclüsselentscheidungen in den Politikfeldern Finanzen, Arbeit und Entstaatlichung, 1982-1998, Opladen, Leske, 2001, p. 71-81.

${ }^{22}$ Archives du ministère des Affaires étrangères français, Europe/Allemagne, 6760, note de l'ambassade de France en RFA, hiver 1986.

${ }^{23}$ Traduction de l'auteur de : « The ETUC recognizes that the interdependence of Community economies is now such that many countries have less freedom to decide unilaterally what demand management policies they should pursue, and that in any event these policies by themselves will not now restore full employment in an acceptable length of time without unacceptable effects on inflation or the balance of payments. » (AN britanniques, «Unemployment: Structural Problems and Policy Implications », note de la CES en vue de la rencontre du 9 février 1977.)

${ }^{24}$ Archives de la CES, 1480, déclaration de la CES, Conseil européen Luxembourg, note du 19 novembre 1980. 
incertains, et ne constituent en aucune mesure une référence en Europe. En 1983 et 1984, le chômage est plus élevé au Royaume-Uni qu'en France ou en Allemagne. La croissance y est supérieure mais cela peut être attribué aux revenus du pétrole de la mer du Nord, qui commencent à affluer. La réélection de Thatcher en 1983 est souvent attribuée au sentiment patriotique né de la guerre des Malouines bien plus qu'à ses résultats économiques. Même au sein de l'administration conservatrice, le doute est souvent de mise. Un officiel constate ainsi en juin 1984 que l'insistance britannique mise sur la libéralisation «contraste dans une certaine mesure avec la pente générale des politiques économiques de certains Etats-membres vers des interventions publiques croissantes ${ }^{25} \gg$. En 1986, le premier livre de Peter Hall, l'un des créateurs de l'école des «variétés de capitalismes » se conclut par une formule prudente : «Les changements induits par Thatcher sont loin d'être permanents ${ }^{26}$ ». Ainsi, si la politique française de relance économique soutenue, et reposant largement sur la consommation, est originale en Europe, y compris au sein de la gauche, elle ne s'oppose pas à une pratique néolibérale dominante car la Grande-Bretagne thatchérienne ne brille pas par ses résultats. Ce sont les difficultés internes à la France qui modifient la donne : en conduisant à une situation très instable, elles menacent en effet de dégénérer en crise financière nationale.

\section{La France, au bord de la crise financière}

Un État est en crise financière lorsqu'il éprouve de grandes difficultés à financer ses dépenses. Pour les décideurs français de 1981-1983, trois contraintes spécifiques doivent être prises en compte ${ }^{27}$. Tout d'abord, le financement des dépenses publiques par le moyen apparemment le plus simple, la création monétaire (solution dite de la planche à billet) est insatisfaisante. Elle est associée, en particulier en Allemagne mais pas seulement, à l'hyperinflation de 1923, ou à la situation de pénurie prévalant avec la création du deutschmark, une monnaie forte et peu inflationniste, en juin 1948. De plus, la période hitlérienne est associée, dès avant le second conflit mondial, à des manipulations monétaires incessantes pour camoufler l'inflation,

25 Traduction de l'auteur de: « Somewhat at odds with the general drift of economic policy in some memberstates towards increased public intervention » (AN britanniques, procédure FOIA, MWB021/1, note P. Martin, 12 juin 1984).

26 Traduction de l'auteur de: « The changes Thatcher has inspired are still far from permanent. » (Peter Hall, Governing the Economy: The Politics of State Intervention in Britain and France, New York, Oxford University Press, 1986, p. 130.)

${ }^{27}$ En général, voir Harold James, International Monetary Cooperation, Washington, International Monteray Fund, 1996. 
notamment par un non-remboursement de certaines dettes et par les privations alimentaires, sans parler des prélèvements sur les populations dominées ${ }^{28}$. L'exemple allemand constitue l'exemple extrême de diffusion d'une mentalité anti-inflationniste dès avant 1973, décennie durant laquelle ces idées se diffusent dans l'espace européen. Des sondages montrent que cette préoccupation touche de nombreux segments de la population occidentale et pas seulement les élites ${ }^{29}$. La CES réclame régulièrement une lutte accrue contre l'inflation après $1973^{30}$. Une inflation trop importante est considérée comme problématique car elle crée une forte incertitude chez l'ensemble des agents économiques, dissuadant l'investissement et la consommation. L'inflation peut affecter le pouvoir d'achat si les revenus n'augmentent pas assez vite, ainsi que l'épargne si les taux d'intérêt réels sont négatifs.

Faute de pouvoir recourir massivement à la création monétaire, car cela entraînerait une inflation importante, la seule solution reste donc d'emprunter, soit en interne auprès de ses concitoyens, soit à l'extérieur, comme c'est le cas de la France pour partie.

Plus généralement, ces contraintes financières s'imposent à tous les pays, capitalistes comme communistes, et ne sont pas le résultat d'un choix idéologique. Les manipulations monétaires destinées à masquer des déficits budgétaires provoquent des conséquences négatives patentes partout, y compris à l'Est. En Pologne par exemple, elles aboutissent à des hausses prix massives pour les produits alimentaires qui ont nourri les troubles de 1980. La République démocratique allemande (RDA) choisit au contraire de subventionner massivement certains produits pour éviter l'inflation, au prix d'un endettement croissant envers l'Ouest, et donc d'une dépendance stratégique ${ }^{31}$.

${ }^{28}$ Frédéric Clavert, Hjalmar Schacht, financier et diplomate, 1930-1950, Bruxelles, Peter Lang, 2009.

29 Stefan Eich et Adam Tooze, «The Great Inflation », in Anselm Doering-Manteuffel, Raphaël Lutz et Thomas Schlemmer (dir.), Vorgeschichte der Gegenwart: Dimensionen des Strukturbruchs nach dem Boom, Göttingen, Vandenhoeck \& Ruprecht, 2016, p. 181.

${ }^{30}$ Archives de la CES, 2066, note envoyée par Walter Braun le 25 octobre 1974 ; 1480, déclaration CES, Conseil européen, Luxembourg, $1^{\text {er }}-2$ décembre 1980, note du 19 novembre 1980.

${ }^{31}$ Christoph Starzec et François Gardes, « Inflation in Poland in the 1970s between Official Figures and the Reality : Virtual Price Approach », et André Steiner, « State Price Policy in the German Democratic Republic in the 1970s and 1980s », in Michel P. Chélini, Laurent Warlouzet (dir.), Slowing Down Prices: European Inflation in the 1970s, Paris, Presses de Sciences Po, p. 191-211 et 85-103 ; Étienne Peyrat et Kristy Ironside, « The Communist World of Public Debt (1917-1991) : The Failure of a Counter-Model ? », in Nicolas Barreyre et Nicolas Delalande (dir.), A World of Debt: The Global Politics of Public Debts from the Late $18^{\text {th }}$ Century, Cambridge, Cambridge University Press, 2018 (à paraître) ; Stephen Kotkin, « The Kiss of Debt : The East Bloc Goes Borrowing », in Niall Ferguson, Charles S. Maier, Erez Manela et Daniel J. Sargent (dir.), The Shock of the Global : The 1970s in Perspective, Cambridge, Harvard University Press, 2010, p. 80-93. 
Un second élément réside dans l'instabilité grandissante du système financier international. La fin du système de Bretton-Woods, entre 1971 et 1976, provoque le passage des cours fixes au cours flottants. Cela se traduit par une forte instabilité des monnaies. Or, un État a plus de mal à convaincre des créanciers extérieurs de lui prêter de l'argent si sa propre monnaie est instable et tend à baisser. Plus généralement, les marchés financiers non régulés gagnent en importance durant cette décennie ${ }^{32}$.

Enfin, le dernier élément à considérer est l'impact financier de la crise économique qui sévit depuis presque une décennie en 1983. Les prix du pétrole ont décuplé en dix ans, produisant un transfert massif de richesse vers les pays exportateurs d'hydrocarbures. Les recettes fiscales diminuent, cependant que les dépenses sociales connaissent une forte progression, encore accentuée par l'augmentation de l'espérance de vie. Se pose alors la question du financement des dépenses sociales, débat lancé en France dès le début des années $1980^{33}$.

La question n'est pas seulement théorique car le Royaume-Uni et l'Italie, deux pays riches et voisins de la France, sortent tous deux de crises financières récentes. Le gouvernement britannique fut touché en 1976. Ses déficits n'étaient pourtant pas massifs mais comme il empruntait largement en monnaie étrangère pour se financer, la baisse de la livre (liée notamment à la forte inflation britannique) augmenta considérablement le coût de refinancement en 1976. En décembre 1976, après de longues négociations, le Royaume-Uni obtint un financement exceptionnel du Fonds monétaire international (FMI) de 2,5 milliards de livres ${ }^{34}$. L'intervention du FMI est donc importante mais pas massive. Son impact est surtout politique. Le Royaume-Uni étant un pays riche et une ancienne grande puissance, recourir au FMI fut une humiliation car l'organisation internationale imposa des coupes budgétaires impopulaires ${ }^{35}$. À partir de ce choc, la politique macro-économique britannique évolua vers une priorité donnée à l'équilibre budgétaire et à la maîtrise de l'inflation.

En Italie, la crise du début de l'année 1977 est tout autant économique et financière que politique. Avec une inflation qui s'établit à $19 \%$ en rythme annuel, une crise politique liée au renforcement du Parti communiste italien qui séduit près de $35 \%$ des électeurs et un déficit

${ }^{32}$ Éric Helleiner, States and the Reemergence of Global Finance : From Bretton Woods to the 1990s, Ithaca, Cornell University Press, 1994.

${ }^{33}$ Pierre Rosanvallon, La Crise de l'État-providence, Paris, Éd. du Seuil, 1981.

${ }^{34}$ Alec Cairncross, The British Economy since 1945, Oxford, Blackwell, 1995, p. 216-217.

${ }^{35}$ Catherine Schenk, The Decline of Sterling : Managing the Retreat of an International Currency, 19451992, Cambridge, Cambridge University Press, 2010, p. 371 et 378. 
budgétaire massif (11\% du PIB), le pays a besoin de soutien international ${ }^{36}$. Il reçoit d'abord un prêt de la CEE puis un prêt du FMI en 1977. En échange, l'institution internationale demande des mesures d'économies. Le plan Pandolfi de 1978 est une concrétisation de cette conversion à des politiques plus austères ${ }^{37}$.

Pour éviter de subir ces contraintes internationales, tous les pays de la CEE, sauf la Grande-Bretagne, ont formé en 1978 le $\mathrm{SME}^{38}$. Celui-ci constitue un engagement à défendre la stabilité des parités monétaires en Europe. Le but est d'éviter toute fluctuation excessive, susceptible de perturber le commerce (notamment par des dévaluations compétitives), le financement des déficits, et de créer une forte incertitude dissuadant les agents de consommer et d'investir. Le SME repose sur l'engagement de chaque État à défendre la parité de sa monnaie, afin de s'assurer qu'elle reste dans la marge de fluctuation étroite. Si les acteurs de marché perdent confiance dans une monnaie et spéculent sur sa baisse, l'État concerné a trois solutions. La première consiste à obtenir le soutien de ses partenaires du SME pour défendre la parité originale, ce qui signifie concrètement un engagement des autres banques centrales européennes à acheter la monnaie visée. La seconde impose de négocier avec ses partenaires un nouveau cours pivot plus bas, ce qui constitue une dévaluation concertée. En pratique, les deux premières solutions sont souvent combinées : une dévaluation concertée est négociée et les États s'engagent à défendre la nouvelle parité en intervenant sur les marchés au besoin. La dernière solution est de sortir du SME, ce qui constitue une humiliation politique, mais aussi un enjeu financier car alors les taux d'intérêt du pays dévalué augmentent.

Ainsi les responsables français se trouvent-ils en 1981-1983 dans un environnement international dégradé. La solidarité du SME continue de protéger la France contre la défiance des marchés, mais les tensions sont vives. En effet, la relance française étant largement fondée sur la consommation, elle se traduit avant tout par une relance des importations. Dans le même temps, les exportations ne progressent pas car les produits français sont peu compétitifs et car les clients de la France subissent toujours la conjoncture déprimée consécutive au

${ }^{36}$ Barbara Stallings, «The IMF in Europe : Inflation fighting in Britain, Italy and Portugal », in Richard Medley (dir.), The Politics of Inflation : A Comparative Analysis, New York, Pergamon Press, 1982, p. 86-90 ; AN britanniques, PREM 16/851, draft record of the European Council, La Haye, 29 novembre 1976.

${ }^{37}$ Francesco Petrini, « The Politics of Inflation and Disinflation : The Italian Case », in Michel-Pierre Chélini et Laurent Warlouzet (dir.), Slowing Prices Down : Adaptation of States and European Economical Actors to the Inflationary Fever in the 1970s, Paris, Presses de Sciences Po, 2016, p. 115-141.

${ }^{38}$ Emmanuel Mourlon-Druol, A Europe made of Money : The Emergence of the European Monetary System, Ithaca, Cornell University Press, 2012. 
second choc pétrolier. Par ailleurs, les mesures de relance augmentent massivement le déficit budgétaire et la dette, le tout dans un contexte de taux d'intérêt élevés qui rendent les emprunts coûteux. Le franc est dévalué à deux reprises, en octobre 1981 et en juin 1982 car les marchés n'ont plus confiance dans cette monnaie assise sur un État qui accumule les déficits. La baisse du franc ne profite pas aux exportations françaises, et a trois conséquences négatives. D'abord, elle augmente le prix des importations, et contribue ainsi à relancer l'inflation. Ensuite, elle augmente les taux d'intérêt car il faut attirer en France des capitaux qui fuient le pays en raison des déficits publics. Enfin, elle rend l'emprunt à l'étranger plus coûteux. Or l'État français doit se financer majoritairement sur les marchés étrangers. Un dernier risque de ces dévaluations à répétition réside dans le déclenchement d'une spirale incontrôlée de dépréciation monétaire cumulative et auto-entretenue. Pour qu'une dévaluation soit efficace, elle doit donc être associée à un plan d'économie budgétaire et de lutte contre l'inflation qui témoigne de la volonté de l'État d'asseoir sa monnaie sur des fondamentaux sains.

En France, la volonté de mener une politique sociale plus économe sur le plan budgétaire est tangible au sommet du pouvoir dès le début, en 1981, et se traduit par la nomination de Jacques Delors comme ministre de l'Économie et des Finances. Dès le 10 juin 1981, il définit l'action gouvernementale en des termes prudents : «rigueur dans la solidarité et vigilance dans la relance $»$, mais il n'est alors pas un poids lourd du gouvernement ${ }^{39}$. La deuxième dévaluation de juin 1982 lui offre une occasion de réagir. Delors adosse la dévaluation à un plan de blocage des prix et des revenus destiné à briser l'inflation (il ne concerne pas le smic, ni le minimum vieillesse ni les allocations familiales), ainsi qu'un plan d'économies visant à limiter le déficit budgétaire à $3 \%$ de la richesse intérieure produite chaque année (le PIB) ${ }^{40}$. La référence à un déficit proche de $3 \%$ date en fait du Conseil des ministres du 15 mars $1982^{41}$. Elle constitue une référence de principe à un déficit modéré. De manière significative, plusieurs responsables de premier plan, Robert Lion, le directeur de cabinet du Premier ministre Pierre Mauroy, et Christian Sautter, secrétaire général adjoint de l’Élysée, font explicitement référence à l'épouvantail de la crise britannique de 1976 dans des documents internes ${ }^{42}$. La crainte de l'humiliation que constituerait un appel au FMI était réelle.

\footnotetext{
${ }^{39}$ L'Année politique, économique et sociale en France : 1981, Paris, Éd. du Moniteur, 1982, p. 399.

${ }^{40}$ L'Année politique, économique et sociale en France : 1982, Paris, Éd. du Moniteur, 1983, p. 513-515.

${ }^{41}$ M. Tracol, La Rigueur et les réformes..., op. cit., p. 530.

42 Ibid., p. 533 ; M. Margairaz, « L’impératif économique », op. cit., p. 340.
} 
Cependant ces mesures se révèlent insuffisantes car la France a du mal à se financer sur les marchés internationaux. Certes, à un peu plus de $3 \%$ du PNB, ses déficits restent relativement modérés surtout si l'on tient compte du fait que des pays comme les États-Unis, le Japon, ou surtout l'Italie ont des déficits bien plus élevés. Les difficultés françaises proviennent d'une combinaison de facteurs externes, comme les taux d'intérêt très élevés, et internes : la dette française augmente massivement entre 1981 et 1983, surtout sa partie flottante ${ }^{43}$. Elle est largement issue de financements extérieurs. De fait, Paris est l'un des principaux emprunteurs mondiaux sur le marché international des capitaux en 1982 et en $1983^{44}$. Le gouvernement utilise le financement par les euromarchés en septembre 1982, puis il parvient à convaincre l'Arabie Saoudite de lui consentir un prêt ${ }^{45}$.

Pourtant, au début de 1983, la situation se dégrade encore en raison du différentiel croissant entre la France et l'Allemagne, tant en termes de besoins de financement que de rythme d'inflation. Sur le plan politique, le contraste entre la victoire électorale de Kohl et la défaite socialiste aux municipales lance la spéculation contre le franc français en mars $1983^{46}$. Une troisième dévaluation en trois ans se profile. Deux possibilités se présentent alors, entre lesquelles Mitterrand hésite. La première option, défendue par de nombreux ministres comme Bérégovoy, Chevènement ou Fabius consiste à dévaluer le franc massivement en sortant du SME ${ }^{47}$. Cette politique devrait se combiner pour les plus radicaux avec un retour au protectionnisme (d'où la dénomination d'« Albanais » associée à certains). Cela fait écho aux réflexions de nombreux experts économiques socialistes ayant préparé l'alternance de 1981, et qui restaient souvent dans un cadre de référence national ${ }^{48}$. L'avantage d'une dévaluation massive serait de rendre les exportations françaises moins

${ }^{43}$ Olivier Feiertag, «Finances publiques, "mur d'argent” et genèse de la libéralisation financière en France de 1981 à 1984 », in S. Berstein et al. (dir.), François Mitterrand..., op. cit., p. 434-437.

${ }^{44}$ Michael Loriaux, France after Hegemony : International Change and Financial Reform, Ithaca, Cornell University Press, 1991, p. 233-235 ; L'Année politique, économique et sociale en France : 1982, op. cit., p. 534.

45 Olivier Feiertag, «La France, le dollar et l'Europe (1981-1989): aux origines globales de l'euro », Histoire@Politique : politique, culture et société, 19, janvier-avril 2013, pp. 128-142, ici p. 135.

${ }^{46}$ L'Année politique, économique et sociale en France : 1982, op. cit., p. 382.

${ }^{47}$ P. Favier, M. Martin Rolland, La décennie Mitterrand. 1, Les ruptures (1981-1984), Paris, Seuil, 1990, p. 441-443; voir notamment le témoignage de Pierre Bérégovoy dans Éric Roussel, François Mitterrand: de l'intime au politique, Paris, Robert Laffont, 2015, p. 410 ; Vincent Duchaussoy, La Banque de France et l'État (1978-1984) : enjeux de pouvoir ou résurgence du mur d'argent?, Paris, L'Harmattan, 2011, p. 104-105.

${ }^{48}$ Mathieu Fulla, Les Socialistes français et l'économie (1944-1981) : une histoire économique du politique, Paris, Presses de Sciences Po, 2016. 
chères et donc plus compétitives. L'optique est donc clairement « néomercantiliste » : l'accent est mis sur le développement industriel y compris par des accrocs au libre-échange international. Cette approche a connu un certain succès mondial dans les années 1970, mais elle ne s'est jamais imposée car la plupart des dirigeants du monde capitaliste restaient marqués par le contre-exemple des années 1930 : la crise de 1929 a été aggravée par le protectionnisme monétaire et commercial, qui, en retour, a contribué à nourrir les dérives autoritaires ${ }^{49}$.

Les opposants à cette voie du repli national développent trois types d'argument. Tout d'abord, si la France adopte le protectionnisme, alors les marchés extérieurs se fermeront aux produits français. Un risque d'escalade de mesures de rétorsion se profile. Par ailleurs, une dévaluation augmente le prix des importations, et donc l'inflation, ce qui risque d'annuler rapidement l'avantage compétitif donné par la dévaluation. Par ailleurs, il faudrait que les producteurs français soient suffisamment compétitifs pour profiter de cette situation. Enfin, une dévaluation massive et non coordonnée avec les partenaires européens risquerait d'aboutir ainsi à une baisse du franc massive et incontrôlée. Cela accentuerait encore les difficultés de financement en monnaie étrangère car il faudrait plus de franc français pour emprunter la même somme en dollar. Les taux d'intérêt risquent d'augmenter très fortement, étranglant ainsi un peu plus les entreprises, les ménages et l'État français, ce qui irait à l'encontre des objectifs de ceux qui veulent sortir du SME précisément pour faire baisser les taux français. Le risque est donc que la France se retrouve obligée de mendier un prêt au FMI.

La seconde option, défendue par Jacques Delors, et par certains conseillers de François Mitterrand, comme Élisabeth Guigou (conseillère pour les relations économiques extérieures) ou Jean-Louis Bianco (secrétaire général à la présidence de la République), consiste à restaurer l'équilibre des finances publiques et à rester dans le SME, faute de quoi la France devra recourir aux aides du FMI, et perdra son indépendance ${ }^{50}$. Ce second camp l'emporte finalement. Si ce débat franco-français est maintenant bien connu, il reste à en considérer la dimension européenne et particulièrement franco-allemande.

\footnotetext{
${ }^{49}$ Sur la persistance de la référence à la crise des années 1930, voir L. Warlouzet, Governing Europe in a Globalizing World..., op. cit., p. 23, 31-33, 79, 142 ; sur le réflexe néomercantiliste, voir ibid., p. 28-32 et 215.

50 P. Favier et M. Martin Rolland, La Décennie Mitterrand..., op. cit., p. 461 ; É. Roussel, François Mitterrand..., op. cit., p. 411-414.
} 


\section{Une résolution franco-allemande}

En mars 1983, l'alternative est claire, entre le protectionnisme et le repli sur soi d'une part, et la voie de la coopération internationale d'autre part. Cette dernière passe par l'obtention d'un engagement allemand (du gouvernement comme de la Banque centrale) à défendre le franc. Cependant, les partenaires de la France ne lui accordent leur soutien qu'en échange de l'adoption de politiques de stabilité efficaces. L'étude des archives montre que cette pression politique se renforce au fur et à mesure des difficultés françaises. Dès la première dévaluation d'octobre 1981, Mitterrand affirme à Schmidt qu'il est résolu à réduire le déficit et à lutter contre l'inflation ${ }^{51}$. Les Allemands apportent leur soutien en intervenant sur les marchés, et en acceptant que la dévaluation française soit incluse dans une opération de réalignement de plus grande ampleur, la plupart des monnaies européennes étant dévaluées contre le deutschmark et le florin néerlandais. Pour Paris, cela permet de relativiser les difficultés françaises et, pour Bonn, cela permet de garder la France dans le jeu européen et de limiter ses tentations protectionnistes ${ }^{52}$. En avril 1982, Helmut Schmidt écrit à Karl-Otto Pöhl, le président de la Bundesbank, à propos du SME. Il y défend un renforcement de la convergence des politiques économiques par une surveillance accrue de la balance des paiements et de la dette extérieure ${ }^{53}$. La situation française est certainement à l'origine de ces préoccupations car Paris dévalue de nouveau en juin 1982. À cette occasion, de nouvelles discussions se tiennent entre Schmidt et Mitterrand ${ }^{54}$. Ce dernier réaffirme son engagement de réduire les déficits et l'inflation mais il peut dorénavant s'appuyer sur un premier et récent train de mesures d'économie et de blocage des prix et salaires. La désindexation des salaires s'impose également. Cette période est considérée comme une rupture par Thomas Piketty car elle marque la fin d'une période débutée en 1968 caractérisée par une progression du salaire minimum supérieure à celle du salaire moyen ${ }^{55}$.

En janvier 1983, le Premier ministre français Pierre Mauroy rencontre le nouveau chancelier Helmut Kohl et lui affirme que la France mène aussi désormais une politique

${ }^{51}$ AAPD, 1981, doc. 290, note sur un entretien Schmidt-Mitterrand in Latché, 8 octobre 1981, p. 1557-1559.

${ }^{52}$ Archives du ministère des Affaires étrangères allemand, ZW/124.417, note AA/412, Boll, 9 octobre 1981.

53 Archives du ministère des Affaires étrangères allemand, ZW/178.868, lettre d'Helmut Schmidt à Karl-Otto Pöhl, 22 avril 1982.

54 AAPD (Akten zur Auswärtigen Politik der Bundesrepublik Deutschland), 1982, doc. 196, note sur des entretiens entre François Mitterrand et Helmut Schmidt, 29 juin 1982, p. 1039-1041.

55 Thomas Piketty, Le Capital au XXI siècle, Paris, Éd. du Seuil, p. 457. 
d' « austérité » ${ }^{56}$. Par ailleurs, les relations sont meilleures entre Mitterrand et Kohl qu'avec son prédécesseur Schmidt. Toutefois, malgré ces démonstrations de bonne volonté, les tensions franco-allemandes sont tangibles en mars 1983, au plus fort des difficultés financières françaises. Le 16 mars, le secrétaire d'État allemand à l'économie Otto Schlecht demande à ce que la prochaine réunion européenne soit consacrée au renforcement de la coordination des politiques économiques, par une lutte accrue contre les déficits budgétaires et contre l'augmentation du coût du travail ${ }^{57}$. La France n'est pas mentionnée mais elle constitue certainement la cible principale. Paris contre-attaque brutalement. Le 19 mars, lors de la réunion des ministres des Finances de la CEE, Jacques Delors refuse toute dévaluation et estime que c'est à la RFA de supporter seule le poids de la réévaluation ${ }^{58}$. Il fait peser le risque d'un retrait de la France du SME et l'adoption de mesures protectionnistes. Cette rhétorique agressive de Delors, pourtant considéré comme un modéré, frappe les dirigeants de la Bundesbank comme en témoignent les documents internes ${ }^{59}$. Cela démontre l'intensité de la crise. En parallèle, Mitterrand demande à Kohl des mesures pour relancer la demande interne. C'est un retour à la théorie de la « locomotive » allemande de $1978^{60}$. L'ambassadeur allemand à Paris se montre très critique envers cette stratégie qui consiste à faire passer la RFA pour un « bouc-émissaire » et conseille de s'y opposer ${ }^{61}$.

Les débats à Bonn sont difficiles. Le 18 mars 1983, lors d'une réunion associant, entreautres, Helmut Kohl, Hans-Dietrich Genscher (ministre des Affaires étrangères), Gerhard Stoltenberg (ministre des Finances) et Karl-Otto Pöhl (Bundesbank), Stoltenberg (qui revient d'un voyage incognito à Paris où il a rencontré les responsables français) insiste sur l'importance d'un geste d'aide de la RFA car il a peur que la menace française de quitter le SME ne soit réelle ${ }^{62}$. Delors lui a confirmé que, pour des raisons politiques, le risque de mesures protectionnistes existait à défaut de mesures allemandes de soutien. Le ministre des

\footnotetext{
${ }^{56}$ AAPD, 1983, note sur des entretiens entre Helmut Kohl et Pierre Mauroy, 20 janvier 1983, p. 72.

57 Archives du ministère des Affaires étrangères allemand, B2/249, lettre de Otto Schlecht à Hans-Werner Lautenschlager, 16 mars 1983

${ }^{58}$ AN britanniques, PREM 19/1027, telex UKREP, 20 mars 1983.

${ }^{59}$ Archives de la Bundesbank, compte rendu des Zentralbankrats des 17 mars et 7 avril 1983.

${ }^{60}$ Archives du ministère des affaires étrangères allemand, B2/249, lettre de Mitterrand à Kohl, 16 mars 1983 ; sur cette tentative de relance concertée derrière la «locomotive allemande»: Laurent Warlouzet, Governing Europe, op. cit., p. 144-5.

${ }^{61}$ AAPD, 1983, doc. 68, télégramme de l'ambassadeur à Paris prénom Herbst, 16 mars 1983.

${ }^{62}$ Archives du ministère des Affaires étrangères allemand, ZW/178.875, note du chef de la chancellerie, 18 mars 1983.
} 
Finances exclut toutefois que le deutschmark supporte seul le coût du réalignement monétaire. Kohl est sur cette ligne. Otto von Lambsdorff défend également le SME, sans doute car il sert à garantir un minimum de discipline. Pöhl est moins pessimiste : une sortie de la France du SME ne serait pas dramatique et, de toute façon, Paris n'a pas intérêt à mener une politique protectionniste. Kohl reste cependant sur une ligne plus européenne : la RFA apportera son soutien à l'opération monétaire mais uniquement si la France consent à des efforts. Le 21 mars 1983, Kohl envoie une lettre à Mitterrand où il souligne l'importance des mesures que la France vient de prendre pour lutter contre l'inflation ${ }^{63}$. Il insiste également sur la nécessité de lever les obstacles aux mouvements des marchandises et des capitaux. Ainsi, la RFA accepte d'aider la France uniquement parce que cette dernière s'engage à prendre des mesures d'austérité et renforce son engagement libre-échangiste ${ }^{64}$. Paris accepte finalement une dévaluation du franc français mais demande en échange une forte réévaluation du deutschmark et un prêt de la CEE ${ }^{65}$.

L'opération monétaire finalement décidée le 21 mars 1983 a une portée européenne : toutes les monnaies sont concernées ${ }^{66}$. Le deutschmark est réévalué de $5,5 \%$, tandis que quatre monnaies sont réévaluées plus modestement, et que trois, dont le franc français, sont dévaluées. Ainsi, les difficultés françaises sont noyées dans une opération européenne. En parallèle, la Bundesbank baisse légèrement ses taux et vend des deutschmarks pour limiter la réévaluation de la monnaie allemande ${ }^{67}$. Enfin, la CEE accorde au même moment, et en complément, un prêt de 4 milliards d'écus à la France, contre le respect du programme d'austérité annoncé à Paris en même temps que la dévaluation. C'est le prêt le plus important accordé par la Communauté dans le cadre de cette procédure d'aide en cas de crise de la balance des paiements ; seules l'Italie et l'Irlande y avait eu recours auparavant ${ }^{68}$. Le viceprésident de la Commission européenne, le Français François-Xavier Ortoli joue un rôle

${ }^{63}$ AAPD, 1983, doc. 110, lettre d'Helmut Kohl à François Mitterrand, 21 avril 1983.

${ }^{64}$ Kenneth Dyson et Kevin Featherstone, The Road to Maastricht : Negotiating Economic and Monetary Union, New York, Oxford University Press, 1999, p. 316-317.

65 AN britanniques, PREM 19/1027, telex UKREP 20 mars 1983 ; PREM 19/1028, brief, economic and social situation, Treasury, 16 mars 1983.

${ }^{66}$ AAPD, 1983, doc. 74, note de Steinkühler, 23 mars 1983.

${ }^{67}$ H. Miard-Delacroix, Le Défi européen de 1963 à nos jours, op. cit., p. 66.

${ }^{68}$ Claude Wolff, Mécanisme des emprunts communautaires destinés au soutien de la balance des paiements des États membres de la Communauté, rapport de la Commission économique et monétaire, Parlement européen, 4 mars 1985 , p. 8 et 14 . 
important pour l'obtention de ce prêt ${ }^{69}$. Malgré les critiques du commissaire européen KarlHeinz Narjes, un chrétien-démocrate allemand, la Commission se montre tolérante sur les mesures de restriction des changes prises par la France en $1983^{70}$. Elle avait adopté la même attitude lorsque la France avait pris des mesures exceptionnelles de défense du franc après Mai $68^{71}$.

Le versant français réside dans une série de mesures d'économies budgétaires annoncée par le ministre de l'Économie et des Finances Jacques Delors le 25 mars ${ }^{72}$. Les taxes sur l'alcool et le tabac sont augmentées cependant qu'un emprunt obligatoire est levé sur les contribuables les plus aisés. Pour éviter les fuites de capitaux, un contrôle des changes strict est établi, cependant que les dépenses des Français à l'étranger sont drastiquement limitées.

Après cette décision, la conversion des élites socialistes à une politique de maîtrise de l'inflation est nette. Certes, des inflexions avaient déjà eu lieu en 1981-1982 car le président Mitterrand menait de concert plusieurs politiques, parfois contradictoires. L'ambiguïté restait de mise. À partir de mars 1983, le choix est net. Les opposants sont marginalisés, comme Jean-Pierre Chevènement qui démissionne justement le 23 mars 1983 pour protester contre cette nouvelle orientation.

Le spectre d'une crise financière en 1983 a convaincu une majorité des élites socialistes que le rapprochement avec la RFA sur le plan monétaire était la seule solution pour diminuer les taux d'intérêt et l'inflation, considérés comme deux préalables à la relance économique. Ainsi, lorsque le problème épineux des montants compensatoires monétaires revient sur le devant de la scène à la fin de l'année 1983, le conseiller agricole du président Mitterrand et futur ministre de l'Agriculture, Henri Nallet, est on ne peut plus explicite : «Le meilleur moyen [de] parvenir [à leur démantèlement] est d'en supprimer la cause, c'est-à-dire

${ }^{69}$ Archives de la Commission européenne, procès-verbal du Conseil, Conseil minefi, 16 mai 1983 ; procèsverbal de la Commission, spécial, 692 session, 11 mai 1983 ; Éric Bussière et Ivo Maes, «Economic and Monetary Affairs : New Challenges and Ambitions », in Éric Bussière et al. (dir.), The European Commission, 1973-86 : History and Memories of An Institution, Bruxelles, Commission européenne, 2014, p. 310 ; Laurence Badel et Éric Bussière (coord.), François-Xavier Ortoli : l'Europe, quel numéro de téléphone, Paris, Descartes et Cie, 2011, p. 185-187.

${ }^{70}$ Archives du ministère des Affaires étrangères français, DECE 1909, note PM, 25 avril 1983.

${ }^{71}$ Laurent Warlouzet, Le Choix de la CEE par la France : les débats économiques de Pierre Mendès France à Charles de Gaulle (1955-1969), Paris, CHEFF, 2011, p. 405-407.

${ }^{72}$ Voir le détail de ce plan dans L'Année politique, économique et sociale en France : 1982, Paris, Éd. du Moniteur, 1983, p. 387-389. 
l'inflation qui est supérieure à celle de notre principal partenaire, l'Allemagne ${ }^{73}$. » Par ailleurs, ce risque de crise financière a contribué à convaincre une partie des responsables socialistes de l'intérêt de la libération des mouvements de capitaux ${ }^{74}$. Le but est d'obtenir des marchés financiers plus liquides, et donc d'éviter au gouvernement français de se retrouver de nouveau face à un risque grave de crise financière en mars 1983, alors même que ses déficits étaient relativement modérés. Le mouvement de conversion à la libération des mouvements de capitaux se produit d'ailleurs à peu près au même moment au FMI ${ }^{75}$.

La crise de 1983 résulte donc bien de difficultés monétaires et financières concrètes, et pas seulement d'un arbitrage politique. Les déficits ne sont pas une vue de l'esprit mais bien une contrainte réelle, comme l'exemple des pays de l'Est le prouve. Le risque de crise financière était réel car l'État français peinait à se financer ${ }^{76}$. Il aurait très bien pu avoir recours au FMI comme le Royaume-Uni en 1976 ou l'Italie en 1977. Cela ne signifie d'ailleurs pas qu'aucune alternative n'était possible, comme l'ont montré la situation de ces deux pays voisins. À l'humiliation aurait pu succéder soit des réformes néolibérales radicales, comme à Londres avec Thatcher à partir de 1979, soit un repli protectionniste mais qui n'aurait sans doute pas empêché la dépendance extérieure, comme l'ont montré la trajectoire des pays d'Europe de l'Est dans les années 1980. Il est également possible d'imaginer une trajectoire italienne d'économie à deux vitesses, dont les déséquilibres sont atténués par un endettement massif.

L'origine des tensions résulte d'un décalage entre les conjonctures de la France d'une part, et de celles de ses principaux partenaires européens d'autre part. Alors que Paris relance, ses voisins restent mirés dans une dépression consécutive au second choc pétrolier. Le clivage est également idéologique, entre une gauche socialiste française séduite par une relance purement nationale et par un réflexe néomercantiliste, face à d'autres élites de la gauche européenne (notamment au SPD et à la CES) qui insèrent leurs réflexions dans le cadre de la mondialisation économique. Certes, la crise française a été accentuée par certains choix radicaux effectués en 1981, notamment la nationalisation à $100 \%$ et le choix d'une relance

${ }^{73}$ AN françaises, 5AG4/EG 34, note d'Henri Nallet, conseiller technique à la présidence de la République, 8 novembre 1983

${ }^{74}$ Cette thèse est au cœur du livre de Rawi Abdelal, Capital Rules : The Construction of Global Finance, Ithaca, Cornell University Press, 2007 ; voir notamment sa discussion dans la section « débat sur un livre » de la revue Monde(s) : histoire, espace, relations, 13, mai 2018.

${ }^{75}$ Jeffrey Chwieroth, Capital Ideas : The IMF and the Rise of Financial Liberalization, Princeton, Princeton University Press, 2010, p. 147-149.

${ }^{76}$ O. Feiertag, «La France, le dollar et l'Europe... », art. cité, p. 18 ; id., « Finances publiques... », art. cité, p. $445-446$. 
largement fondée sur la consommation. Cependant ces choix n'étaient pas forcément incohérents en mai 1981, alors que la politique industrielle du gouvernement précédent n'avait pas donné satisfaction. Par ailleurs, la rigueur barriste offrait de confortables marges de manœuvres financières, la France étant alors en meilleure posture que l'Allemagne fédérale.

La crise s'est résolue dans un cadre européen, moins du fait de la Grande-Bretagne thatchérienne (dont les résultats économiques ne sont pas meilleurs que ceux du continent), que de par l'influence du gouvernement ouest-allemand, tant du social-démocrate Schmidt que du chrétien-démocrate Kohl. Tous deux restent ancrés dans la culture anti-inflationniste allemande, nourrie par une histoire tourmentée. Bonn maintient un dialogue constant avec Paris pour associer assistance monétaire et réformes structurelles en France. La relation franco-allemande s'articule avec la dimension européenne du SME, même si la Commission européenne joue alors un rôle relativement secondaire. Les élites socialistes françaises font un choix conscient et affirmé de convergence vers les politiques de stabilité prônées par l'Allemagne. Ainsi, la référence des $3 \%$ de déficit public est-elle adoptée dès 1982 car elle traduit un objectif susceptible de ne pas entraîner de problème de financement des dépenses de l'État. En mars 1983, la volonté de rester dans le SME et de conforter l'axe francoallemand est considérée comme une solution rationnelle pour baisser les taux d'intérêt, et ainsi faciliter le financement tant de l'État que de l'économie française. Plus que d'un tournant «néolibéral» (selon le sens défini en introduction), il s'agit d'un retour à l'orthodoxie budgétaire destinée à pérenniser les avancées sociales du début du septennat, à défaut de pouvoir les renforcer comme cela était prévu initialement. L'orientation est libreéchangiste plus que néolibérale.

La résolution du problème financier entraîne une rupture forte sur le plan international avec la confirmation sans équivoque du choix de l'Europe comme voix de l'influence mondiale, alors qu'une incertitude persistait dans ce domaine. À défaut de l'adhésion à une politique de stabilité, l'intervention du FMI aurait pu conduire à une humiliation française, et à un déclassement inédit depuis la restauration du crédit international de la France par le plan Rueff mis en œuvre par Charles de Gaulle à la fin de l'année 1958. Dans les deux cas, pour De Gaulle comme pour Mitterrand, la dévaluation et le rétablissement des équilibres budgétaires ont été guidés par une volonté d'influence mondiale, qui passe par un renforcement de la coopération européenne. À chaque fois, la rigueur conditionne la grandeur. 
Laurent Warlouzet est professeur d'histoire à l'Université du Littoral-Côte d'Opale (ULCO). Agrégé d'histoire, il a été postdoctorant à l'Institut universitaire européen de Florence (IUE/EUI) et à la London School of Econnomics and Political Science (LSE). Spécialiste d'histoire de l'Europe occidentale (France, Royaume-Uni, Allemagne, Union européenne), en particulier sous l'angle des politiques économiques et sociales, il vient de publier : Laurent Warlouzet, Governing Europe in a Globalizing World : Neoliberalism and its Alternatives following the 1973 Oil Crisis (Routledge, 2018). 